\section{Pediatric Intensive Care Unit admission criteria for haemato- oncological patients: a basis for clinical guidelines implementation}

\author{
Marco Piastra, ${ }^{1}$ Giuliana Fognani, 2 \\ Alessia Franceschi3 \\ On Behalf Of The The "ICARO Italian \\ Network For Intensive Care In Pediatric \\ Oncology" \\ 1PICU, Emengency Department, Catholic \\ University A. Gemelli Hospital, Rome; \\ 2PICU, A. Meyer Children Hospital, \\ Florence; \\ 3PICU, G.Gaslini Children Hospital, \\ Genua, Italy
}

\section{Abstract}

Recent advances in supportive care and progress in the development and use of chemotherapy have considerably improved the prognosis of many children with malignancy, thus the need for intensive care admission and management is increasing, reaching about $40 \%$ of patients throughout the disease course. Cancer remains a major death cause in children, though outcomes have considerably improved over the past decades. Prediction of outcome for children with cancer in Pediatric Intensive Care Unit (PICU) obviously requires clinical guidelines, and these are not well defined, as well as admission criteria. Major determinants of negative outcomes remain severe sepsis/septic shock association and respiratory failure, deserving specific approach in children with cancer, particularly those receiving a bone marrow transplantation. A nationwide consensus should be achieved among pediatric intensivists and oncologists regarding the threshold clinical conditions requiring Intensive Care Unit (ICU) admission as well as specific critical care protocols. As demonstrated for the critically ill non-oncologic child, it appears unreasonable that pediatric patients with malignancy can be admitted to an adult Intensive Care Unit ICU. On a national basis a pool of refecence institutions should be identified and early referral to an oncologic PICU is warranted.

\section{Introduction}

Cancer remains a major death cause in children, though outcomes have considerably improved over the past decades. ${ }^{1-3}$ Outcomes for children diagnosed with cancer have changed since ' 70 from $80 \%$ mortality to $80 \%$ survival; 2 while children with solid tumors 5 year survival has been reported as $67 \%$, cure rates for childhood leukemia now approach 90\%.4,5 Moreover, hematopoietic stem cell transplantation (HSCT) indications are still expanding for both malignant and non-malignant diseases.

Some of the improvement in survival has been linked to more aggressive cancer treatment regimens. However, these protocols are associated with an increase in complications and life-threatening events that may require PICU admission. As a consequence, cancer therapy advances have resulted in an increased need for critical care services for these children. Intensive care itself can cause unavoidalble complications, the most prominent of which are infections that result from treatment-associated immunosuppression. Eventually, nearly $40 \%$ of pediatric cancer patients require intensive care services, accounting for approximately $3 \%$ of all PICU admissions. 6,7

Recently, a 3-year (2003-2005) nationwide italian (involving 27 institutions) survey, regarding the outcomes of cancer children outlined respiratory failure and severe sepsis/septic shock as major risk factors for cancer-related ICU mortality (M.Piastra, unpublished data). Globally, 1367 patients were enrolled, of whom 464 medical (non-postoperative) patiens; PICU survival was about 71.5\%, with substantial differences among different critical illnesses. Before these data become available, national survival rates for PICU cancer patients were not known. In the past decades reported survival rates ranged from almost $100 \%$ mortality in patients requiring prolonged mechanical ventilation to $100 \%$ survival in those receiving uncomplicated postoperative care; therefore, concerns arose regarding the futility of treating critically ill cancer patients. ${ }^{8-11}$ In fact, the few published studies on the outcome of children with malignancies who were admitted to PICU reported poor outcomes, especially for those requiring ventilatory support or inotropic support in the context of sepsis or after bone marrow transplant.12,13 Nevertheless, recent studies have demonstrated promising outcomes with an overall survival rate of $>80 \%$ in paediatric cancer patients admitted to a PICU.6,14 Furthermore, great efforts have been undertaken to assess the risk of mortality and outcome in paediatric cancer patients. $^{15}$

Taking into consideration recent italian epidemiological data, an effort has been undertaken in order to develop and disclose PICU admission criteria for onco-hematological children. Based on main causes, baseline suggestions are being offered aimed at early referral of critically ill patients affected by systemic infections and respiratory failure.
Correspondence: Marco Piastra, Terapia Intensiva Pediatrica, DEA Policlinico Universitario A. Gemelli, Università Cattolica del Sacro Cuore, L.go A. Gemelli 8, 00168 Roma, Italy. Tel. +39.06.30155203 - Fax. +39.06.30155283.

E-mail: marco_piastra@yahoo.it

Key words: intensive care, malignancy, Pediatric Intensive Care Unit admission criteria, children, critically ill.

Acknowledgments: ICAR0 steering committee : M. Piastra, D. Pietrini(UCCS, Roma), A.Tornesello (Lecce), P. Ingelmo (Monza), F. Locatelli (OPBG, Roma), A. Pession (Bologna), P. Cogo (OPBG, Roma), S. Cesaro (Verona). ICARO group: ( complete list of study participants, not in alphabetical order, with their location in brackets): A. Conio, R. Grossetti (Torino), A. Franceschi, G. Ottonello (Genova), C. Gallini, F. Racca (Alessandria), F.Ferrero (Novara), E. Calderini, G. Chidini (Milano), A. Wolfler, I. Salvo (Milano), M. Lamperti (Milano), D. Codazzi (Bergamo), R. Fumagalli (Monza), M.G. Molinaro (Brescia), L. Carnevale (Pavia), A. Pettenazzo (Padova), P. Biban (Verona), S. Furlan, F. Savron (Trieste), R. Pagni (Ancona), S. Baroncini (Bologna), M. Calamandrei, G. Fognani (Firenze), M. Marano, F. Stoppa, N. Pirozzi, F. Chiusolo (OPBG,Roma), D. De Luca, E. Stival, L. Marzano, F. Caliandro, G. Conti (UCSC,Roma), R. Testa, G. Savoia (Napoli), A. Guddo (Palermo), E. Gitto (Messina), G. Melchionda, A. Del Gaudio (SG Rotondo, FG), R. Caione (Lecce).

Received for publication: 29 November 2010. Accepted for publication: 19 April 2011.

This work is licensed under a Creative Commons Attribution 3.0 License (by-nc 3.0).

(C) Copyright M. Piastra et al., 2011

Licensee PAGEPress, Italy

Pediatric Reports 2011; 3:e13

doi:10.4081/pr.2011.e13

\section{Severe sepsis/septic shock patients}

For severe sepsis-septic shock the observed mortality remains still high, and consistently higher than recently reported for the italian non-oncologic pediatric population: namely $55 \%$ in the above mentioned italian survey. 16 International sepsis guidelines advocate a timely approach to cardiovascular derangements in septic patients, involving early monitoring of hemodynamic status for patients with fluid-refractory shock. Self-reported data evidenced that advanced continuous hemodynamic monitoring has been reported by a minority of institutions in the 2003-2005 survey. A limited use of invasive monitoring in children and 
infants should not be longer accepted, given the possibility of accurate and safer echo-guided techniques even in younger infants. 17 According to recent international guidelines, 18 hemodynamic monitoring should guide the management of critically ill children very early after ICU admission: the rapid identification of the hemodynamic pattern and - as a consequence -preload/vasoactive combination therapy tailoring can really influence the mortality from sepsis also in cancer children.

The timely identification of sepsis towards severe sepsis-septic shock shift and the early ICU referral of these patients is of mainstem importance. The widespread use of vasoactive agents outside an ICU setting should be discouraged, lacking appropriate hemodynamic monitoring; conversely, educational programs should be implemented in order to improve early circulatory status diagnosis. Both noninvasive Doppler devices and bedside basic echocardiography can be introduced, having an acceptable learning curve - thereby increasing diagnostic accuracy yet in the non-intensive setting.

PICU referral of severely hypotensive (late referral) patients should be prevented; patients with unexplained tachycardia (having ruled out fever and agitation) and early diastolic blood pressure decrease or signs of hypoperfusion (including increasing negative Base Excess or hyperlactacidemia) are more likely to benefit from PICU admission (Table 1).

\section{Acute respiratory failure}

A recent review paper on pediatric oncology patients requiring conventional respiratory support outlined current outcomes both in Bone Marrow Transplantation (BMT) and nonBMT children, 19 while encouraging clinical research regarding both NIV and new interfaces suitable for pediatric use, Long-term survival after even prolonged mechanical ventilation in PICU has been reported. Very recently, an italian pediatric experience ${ }^{20}$ seems to confirm the role of NPPV in immunocompromised/cancer children affected by early Acute Respiratory Ditress Syndrome (ARDS), as yet demonstrated and well established in adults. ${ }^{21-}$ 24 In this feasibility study we reported that $56 \%$ of immunocompromised children with ARDS, deemed to require conventional invasive ventilation, could be successfully managed with Non invasive Positive Pressure Ventilation (NPPV), avoiding endotracheal intubation and possibly related complications. ${ }^{20}$ Children successfully ventilated with NPPV also showed a shorter PICU and hospital stay, a lower incidence of septic complications, including

Table 1. Organ failure signs triggering Intensive Care Unit team assessment and possibly Intensive Care Unit admission/treatment in $\mathrm{H} \& \mathrm{O}$ pediatric patient.

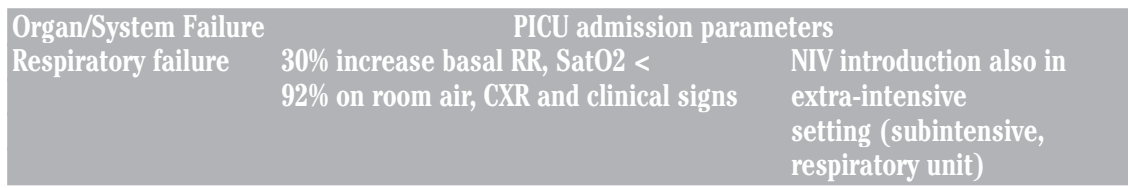

\begin{tabular}{lccc} 
Severe sepsis & \multicolumn{4}{c}{ Haemodynamic compromise signs according to age: threshold values } \\
& Diastolic BP & Systolic BP & Heart Rate \\
Infant & $53 \mathrm{mmHg}$ & $72 \mathrm{mmHg}$ & 180 \\
Preschool & $53 \mathrm{mmHg}$ & $7 \mathrm{mmHg}$ & 160 \\
School & $57 \mathrm{mmHg}$ & $83 \mathrm{mmHg}$ & 140 \\
Adolescent & $66 \mathrm{mmHg}$ & $90 \mathrm{mmHg}$ & 125
\end{tabular}

Neurologic compromise GCS $<12$ or $>3$ points variation from baseline; relapsing seizures

Kidney failure $\quad$ Fluid overload, oliguria, electrolyte derangements, CRRT (all)

Liver failure Severe hypocoagulability, liver support, hepatic enkephalopathy

BP, blood pressure; CRRT, continuous renal replacement therapy; CXR, chest X-rays; GCS, glasgow coma scale; NIV, non invasive ventilation; $\mathrm{RR}$, respiratory rate

Ventilator Associated Pneumonia (VAP) and septic shock. Notably, at PICU admission severity scores and organ failures did not differ between the NPPV-success and failure groups. As a whole, these data strongly suggest that a NPPV trial should be considered in immunocompromised children with early Acute Lung Injury (ALI) and ARDS.

Moreover, recent data from a randomized trial seems to sustain an even earlier introduction of noninvasive ventilatory support. In his paper, VM Ranieri and his coworkers highlinght the importance of very early Continous Positive Airway Pressure (CPAP) introduction: this study demonstrates that early use of CPAP in the hematological ward in immunosuppressed patients with hematological malignancy, but without a secure diagnosis of infection, may prevent evolution of respiratory symptoms to acute lung injury requiring ventilatory support and ICU admission. ${ }^{25}$ These experiences should be confirmed even in childhood, but it seems that a similar rationale exists. Based on these data and the available scientific evidence, an erlier CPAP introduction in respiratory distressed patients should be encouraged, compared to previous recommendations. The introduction of CPAP within the pediatric oncology setting represents an interesting option that can be performed provided that i) a continuous gas exchange monitoring can be guaranteed; ii) both medical and non-medical caregivers have been specifically trained iii) a regular supervision and timely intervention by pediatric ICU team can be offered. Previously, respiratory parameters threshold had been identifyied for PICU referral (or respiratory support introduction, depending on the setting): the association of Sat02 $\geq 92 \%$ while on $0.30 \mathrm{FiO} 2$ has been suggested. By the light of recent evidences, it seems that every respiratory change (in associ- ation with chest film alterations) including Respiratory Rate increase by $30 \%$ and SatO2 $<92 \%$ on room air should be investigated and early CPAP implementation considered. We actually agree with such a more interventional attitude, thus we will increasingly offer CPAP for hypoxaemic $\mathrm{H} \& 0$ patients than simple oxygen support in the future.

\section{Pediatric oncology intensive care specificity}

As recently demonstrated, intensive care outcomes can be very different outside a pediatric-devoted ICU: in fact, a suboptimal outcome of children in general adult ICUs in Italy is suggested by this analysis. ${ }^{26}$ Interestingly, the time frame is overlapping with our epidemiological study (2003-2005), involving patients from 27 italian ICUs (21 PICUs and 6 general ICUs). As a general rule, some reluctance or unjustified delay in treating pediatric oncology critically ill patients can be present in general ICUs. Again, a strong rationale supporting the centralisation of critically ill children to PICUs exist, particurlarly in the setting of pediatric oncology. In fact, early invasive/noninvasive hemodynamic and ventilatory support may really impact on the outcome of the cancer pediatric patient. Patients should be rapidly referred - based on pediatric intensivist's opinion- from areas lacking specialised pediatric intensive care. Recent advances in pediatric oncology clearly require a high-level critical care as a part of the therapeutic program. Therefore, a network of specialised oncology critical care institutions should be identifyied on a national basis. 
Med 1991;17:11-5.

\section{References}

1. Waskerwitz MJ, Ruccione K. An overview of cancer in children in the 1980s. Nurs Clin North Am 1985;20:5-29.

2. Bleyer WA. Epidemiologic impact of children with brain tumors. Childs Nerv Syst 1999;15:758-763.

3. Bleyer WA. The US Pediatric cancer clinical trials programmes: International implications and the way forward. Eur J Cancer 1997;33:1439-47.

4. Friedmann AM, Weinstein HJ. The role of prognostic features in the treatment of childhood acute lymphoblastic leukemia. Oncologist 2000;5:321-8.

5. Creutzig U, Zimmermann M, Ritter J, et al. Definition of a standard-risk group in children with AML. Br J Haematol 1999;104: 630-9.

6. Dalton HJ, Slonim AD, Pollack MM. Multicenter outcome of pediatric oncology patients requiring intensive care. Pediatr Hematol Oncol 2003;20:643-9.

7. Rosenman MB, Vik T, Hui SL, et al. Hospital resource utilization in childhood cancer. J Pediatr Hematol Oncol 2005;27: 295-300.

8. Butt W, Barker G, Walker C, et al. Outcome of children with hematologic malignancy who are admitted to an intensive care unit. Crit Care Med 1988;16:761-4.

9. Meert K, Lieh-Lai M, Sarnaik I, Sarnaik A. The role of intensive care in managing childhood cancer. Am J Clin Oncol 1991; 14:379-82.

10. Sivan Y, Schwartz PH, Schonfeld T, et al. Outcome of oncology patients in the pediatric intensive care unit. Intensive Care
11. Heney D, Lewis IJ, Lockwood L, et al. The intensive care unit in pediatric oncology. Arch Dis Child 1992;67:294-8.

12. Hallahan AR, Shaw PJ, Rowell G, et al. Improved outcomes of children with malignancy admitted to a pediatric intensive care unit. Crit Care Med 2000;28:3718-21.

13. Heying R, Schneider DT, Korholz D, et al. Efficacy and outcome of intensive care in pediatric oncologic patients. Crit Care Med 2001;29:2276-80.

14. Keengwe IN, Stansfield F, Eden 0, et al. Paediatric oncology and intensive care treatments: changing trends. Arch Dis Child 1999;80:553-5.

15. Schneider DT, Lemburg P, Sprock I, et al. Introduction of the oncological pediatric risk of mortality score (O-PRISM) for ICU support following stem cell transplantation in children. Bone Marrow Transplant 2000; 25:1079-86.

16. Wolfler A, Silvani P, Musicco M, Et al. Italian Pediatric Sepsis Study (SISPe) group. Incidence of and mortality due to sepsis, severe sepsis and septic shock in Italian Pediatric Intensive Care Units: a prospective national survey Intensive Care Med 2008;34:1690-7.

17. Pietrini D, Piastra M, Lamperti M, Ingelmo PM. New trends in pediatric anesthesia. Minerva Anestesiol 2009;75:191-9.

18. Brierley J, Carcillo JA, Choong K, et al. Clinical practice parameters for hemodynamic support of pediatric and neonatal septic shock: 2007 update from the American College of Critical Care Medicine. Crit Care Med 2009;37:666-88.

19. Tamburro RF, Barfield RC, Shaffer ML, et al. Changes in outcomes (1996-2004) for pediatric oncology and HSCT patients requiring invasive mechanical ventilation Pediatr Crit Care Med 2008;9:270-7.

20. Piastra M, De Luca D, Pietrini D, et al. Noninvasive pressure support ventilation in immuno-compromised children with ARDS: a feasibility study. Intensive Care Med 2009;35:1420-7.

21. Hilbert G, Gruson D, Vargas F, et al. Non invasive ventilation in immunosuppressed patients with pulmonary infiltrates, fever, and acute respiratory failure. N Engl J Med 2001;344:481-7.

22. Antonelli M, Conti G, Bufi M, et al. Noninvasive ventilation for the treatment of acute respiratory failure in patients undergoing solid organ transplantation: a randomized trial. JAMA 2000;283:235-41.

23. Conti G, Marino P, Cogliati A, et al. Noninvasive ventilation for the treatment of acute respiratory failure in patients with hematological malignancies: a pilot study. Intensive Care Med 1998;24:128388.

24. Rocco M, Conti G, Antonelli M, et al. Noninvasive pressure support ventilation in patients with acute respiratory failure after bilateral lung transplantation. Intensive Care Med 2001;27:1622-6.

25. Squadrone V, Massaia M, Bruno B, et al. Early CPAP prevents evolution of acute lung injury in patients with hematologic malignancy Intensive Care Med 2010;36: 1666-74.

26. Cogo PE, Poole D, Codazzi D, et al. Outcome of children admitted to adult intensive care units in Italy between 2003 and 2007. Intensive Care Med 2010;36: 1403-09. 